\title{
Observations of ionospheric escape on Venus' nightside
}

\author{
J. D. Mihalov, ${ }^{1}$ C. T. Russell, ${ }^{2}$ W. T. Kasprzak, ${ }^{3}$ and W. C. Knudsen ${ }^{4}$
}

\begin{abstract}
A population of low-energy $(0-250 \mathrm{~V} E / q)$ ions with tailward directed velocity vectors and energies above that for escape from Venus is evident in nightside data from the Ames plasma analyzer on the Pioneer Venus Orbiter spacecraft. Good correlations with solar wind parameters were not obtained for the magnitudes of these ion fluxes, but tendencies for occurrence at times of tailward oriented magnetic fields and for alignment of the ion flows with the magnetic field were found. These tendencies seemed to be enhanced for higher-energy ions. In a few cases where comparisons were made, the ion fluxes were consistent with simultaneous $\mathrm{O}^{+}$measurements by the neutral mass spectrometer experiment on the spacecraft. The mean flux observed of the escaping nightside ions, averaged over an approximately 10 -week-long spacecraft nightside season, was $<2 \times 10^{6} \mathrm{~cm}^{-2} \mathrm{~s}^{-1}$.
\end{abstract}

\section{Introduction}

Extensive in situ measurements in Venus' magnetic tail and nightside ionosphere have been acquired by the Pioneer Venus Orbiter (PVO) [Colin, 1980], from December 1978 [cf. Brace and Kliore, 1991; and Phillips and McComas, 1991] to October 1992. The PVO orbit had a near-polar inclination, with a 12 Venus radii apoapsis (24-hour period). Analysis of the nightside observations revealed many previously unknown features, for example, the striking plasma and magnetic field configurations [Brace et al., 1987], in which filaments of high-beta plasma extend downstream at high altitudes (the ionotail), and the remarkable temperature, density, and solar wind dependences on the nightside [Brace et al., 1990; Knudsen et al., 1987].

Kasprzak et al. [1982, 1987, 1991] have observed escaping superthermal ( $>40 \mathrm{eV}$ ) ionospheric ions on the night side of Venus. These were principally $\mathrm{O}^{+}$. The average flux was about $10^{5} \mathrm{~cm}^{-2} \mathrm{~s}^{-1}$. While the rate of loss of oxygen from Venus' atmosphere is an important parameter when the atmospheric evolution with time is considered [Hunten et al., 1989], this superthermal ion escape is much lower than other reported loss fluxes [Brace et al., 1987; Kasprzak et al., 1991]. Intriligator et al. [1994] have presented and discussed an $\mathrm{O}_{2}{ }^{+}$detection at $-1100 \mathrm{~km}$ altitude, on Venus' nightside during June, 1992.

Low-energy $(0-250 \mathrm{VE} / q)$ ion fluxes were measured by the Ames plasma analyzer, during 10 nightside periapsis "seasons," from 1981 to 1992. These seasons occurred once each 224.7 day Venus year. During its nearly 14-year orbital lifetime the PVO periapsis first rose and then fell as a result of solar gravitational perturbations, which eventually forced the

\footnotetext{
'Planetary Systems Branch, NASA-Ames Research Center, Moffett Field, California

${ }^{2}$ Institute for Geophysics and Planetary Physics, UCLA, Los Angeles.

${ }^{3}$ NASA-Goddard Space Flight Center, Greenbelt, Maryland

${ }^{4}$ Knudsen Geophysical Research, Monte Sereno, California

Copyright 1995 by the American Geophysical Union.

Paper number $95 \mathrm{JA0} 1342$.

0148-0227/95/95JA-01342\$05.00
}

spacecraft to enter the atmosphere where it was destroyed [Strangeway, 1993]. In addition to the 10 seasons of lowenergy data it was possible to extend the set of measurements with additional data at energies above $50 \mathrm{~V} \mathrm{E/q}$ from two seasons at the highest periapsis altitudes. These 12 nightside seasons span all the periapsis altitudes but are not contiguous in time. On Table 1, the periapsis altitudes for the 10 of the 12 seasons with periapses above $700 \mathrm{~km}$ are given, as well as the dates when data could be acquired during each season, and the associated sunspot number $R_{l}$, which is tabulated as a measure of solar activity. For periapsis altitudes above $\sim 1000 \mathrm{~km}$, a population of ions with tailward directed velocity vectors and energies above that for escape from the planet can be distinguished. In this paper, broad characteristics of these escaping ions are presented, as are various correlations of the escaping ion fluxes, and the identification of the ion species is addressed by comparison with measurements of specific ion species, from other Pioneer Venus Orbiter plasma experiments.

\section{Experiment and Data Acquisition}

The solar wind plasma analyzer (OPA) [Intriligator et al., 1980] on the Pioneer Venus Orbiter spacecraft used quadrispherical, $90^{\circ}$ electrostatic deflection plates, and multiple (five) current collectors, with electrometer tubes for current measurement. The view direction was normal to the spacecraft spin axis so that nearly $4 \pi$ sr solid angle could be sampled during one spacecraft revolution. Three energy ranges were commandable, for solar wind plasma (ions), electrons, and "low-energy" ions. In addition, there were selectable choices for a few different temporal sampling schemes.

Usually, low-energy ion data $(0-250 \mathrm{~V} \mathrm{E} / q)$ were acquired on every third nightside periapsis orbit. This is illustrated by Figure 1, which shows the periods for nightside season 6 during which the plasma analyzer was actually configured for low-energy ion measurements. Times when a threshold flux $\left(4.8 \times 10^{5} \mathrm{~cm}^{-2} \mathrm{~s}^{-1}\right.$, cold beam) was exceeded $(24 \%$ of the total samples) are indicated as well (small circles). As indicated 
in the Figure 1, during this season it was actually possible to acquire low-energy ion data on nearly every third orbit; there are only two exceptions.

In addition, out of the total time that the spacecraft spent on Venus' nightside, usually about as large a fraction was spent

Table 1. PVO Nightside Seasons

\begin{tabular}{|c|c|c|c|}
\hline $\begin{array}{l}\text { Nightside } \\
\text { Season } \\
\text { Number }\end{array}$ & Dates & $\begin{array}{l}\text { Periapsis } \\
\text { Altitudes, } \\
\text { km }\end{array}$ & $\begin{array}{l}\text { Sunspot } \\
\text { Number } \\
R_{I}\end{array}$ \\
\hline 5 & June 24 to Sept. 7, 1981 & $715-896$ & 153 \\
\hline 6 & Feb. 7 to April 17, 1982 & $1052-1205$ & 145 \\
\hline 7 & $\begin{array}{l}\text { Sept. } 23 \text { to Oct. } 11 \text {, and } \\
\text { Nov. } 22 \text { to } 25,1982\end{array}$ & $1357-1483$ & 110 \\
\hline 8 & May 9 to July 8, 1983 & $1623-1729$ & 92 \\
\hline 9 & Dec. 17,1983 to Feb. 15,1984 & $1834-1924$ & 56 \\
\hline 10 & July 31 to Sept. 26, 1984 & $2005-2077$ & 22 \\
\hline 11 & March 14 to April 16, 1985 & $2146-2205$ & 18 \\
\hline 19 & Feb. 9 to April 16, 1990 & $1502-1379$ & 137 \\
\hline 20 & $\begin{array}{l}\text { Sept. } 20 \text { to Oct. } 18, \text { and } \\
\text { Nov. } 19 \text { to } 30,1990\end{array}$ & $1237-1090$ & 144 \\
\hline 21 & May 1 to July 9, 1991 & $928-760$ & 154 \\
\hline
\end{tabular}

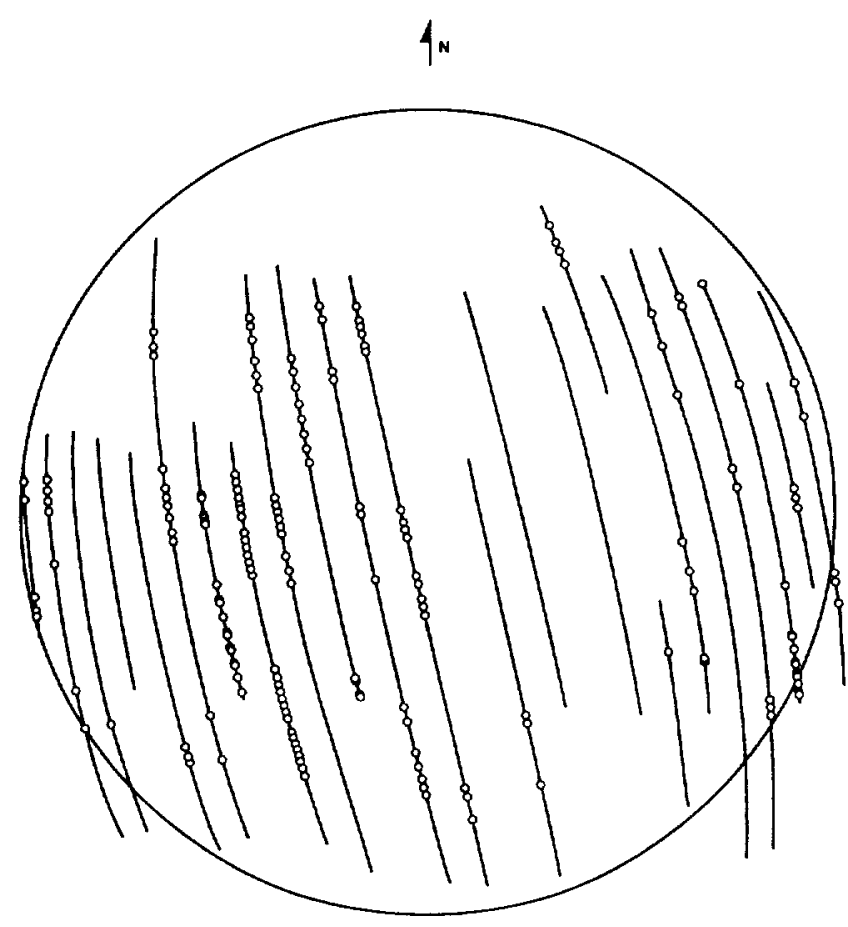

- Low energy lon reaponae above threehold

Figure 1. Example of data collection schedule for the lowenergy ion mode of the plasma analyzer, for nightside periapsis season 6 . The view is toward the Sun, from behind Venus. The low-energy ion mode was used near periapsis on every third orbit, as indicated by the portions of the orbits shown. A threshold was selected to be above instrumental noise. Locations of responses that were above the threshold are indicated (small circles; $-24 \%$ of all the samples). with the analyzer configured to measure electron fluxes (every third orbit, again) as was spent to measure low-energy ion fluxes. The remaining time, bigher-energy ions $(50-8000 \mathrm{~V}$ $E / q$ ) were measured; that data overlaps the lower-energy set, in energy, and can be combined with it to increase statistics for some purposes. The maximum time in Venus' shadow, for any orbit during this season, is $21 \mathrm{~min}$ (orbits near the center of Figure 1), the periapsis passages are from north to south, and successive orbits march across Figure 1 from left to right.

\section{Results}

Figure 2 gives the energy (per unit charge) and azimuthal direction of all low energy ion fluxes from nightside periapsis seasons 5 and 11, respectively, observed between various
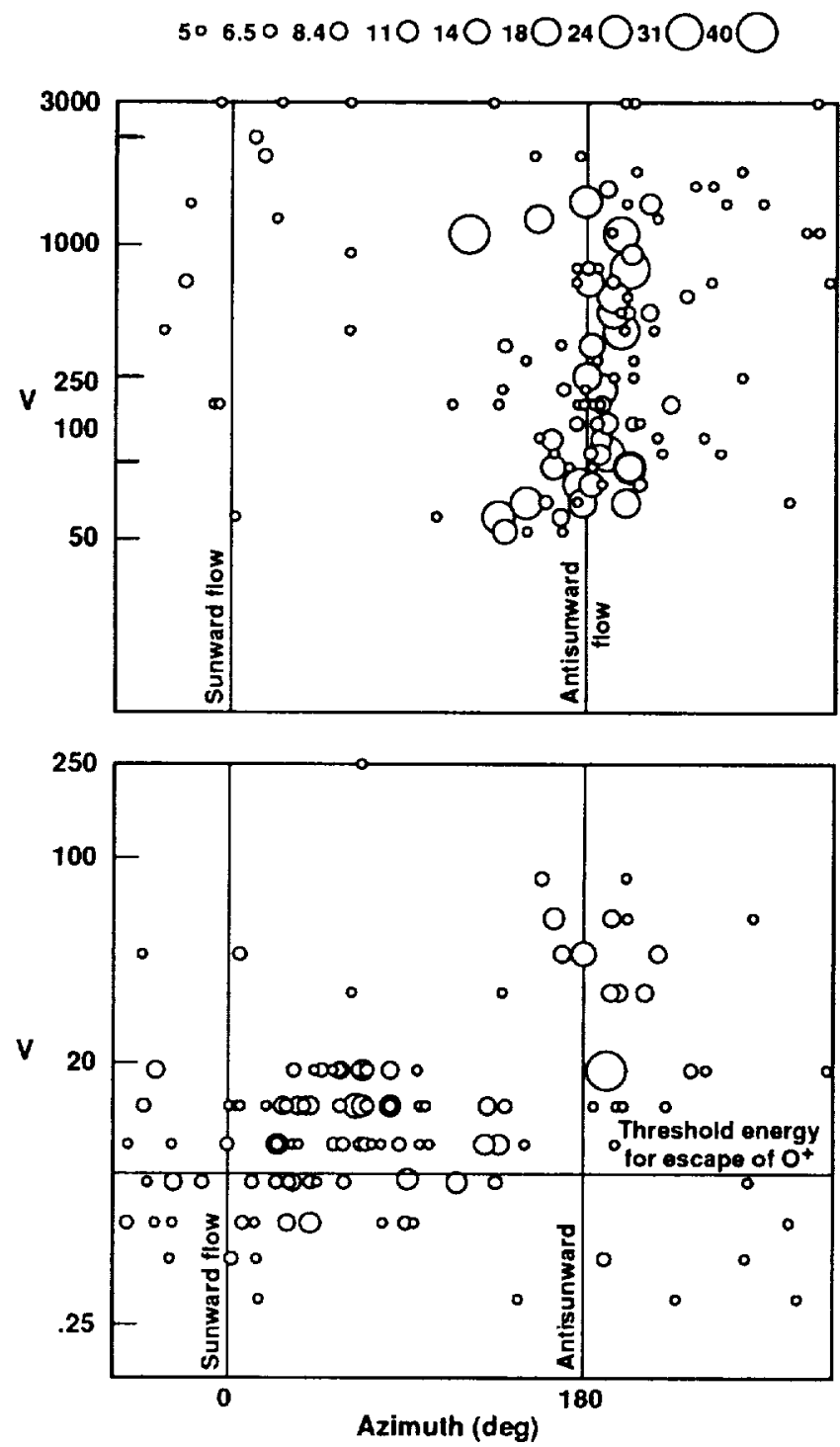

Figure 2. Low-energy ion response azimuth (sector number) versus energy per unit charge, above a noise threshold, for nightside season 5 (lower), and 11 (upper). The numbers indicate threshold cold beam fluxes, in units of $10^{5} \mathrm{~cm}^{-2} \mathrm{~s}^{-1}$, for the various symbols. Lower azimuth angles indicate flow from the east. 
thresholds, as indicated. The lowest threshold is intended to eliminate responses due to noise. These two seasons span the ionotail altitude range, above the main nightside ionosphere, that was sampled at periapsis. For season 11, the "low-energy" mode was not used. The nine flux level increments are each about $30 \%$ wide. For the vast majority of the ion fluxes, the measured polar direction of flow is northward, which corresponds to the southward motion of the spacecraft. Consequently, the polar angular resolution of these flow measurements is not high, since the poleward current collectors of the experiment are the widest of the set, each 47 deg wide. This, in turn, introduces an uncertainty in the nonpolar speed component of $- \pm 30 \%$.

For season 5, responses in the left-hand portion of Figure 2 appear to correspond with motion of the spacecraft through the nearly stationary $\mathrm{O}^{+}$plasma [Brace et al., 1983] of Venus' nightside upper ionosphere, as these responses seem to be bounded near the energy that corresponds to $\mathrm{O}^{+}$with the speed of the spacecraft's (southward) motion, $9.1 \mathrm{~km} / \mathrm{s}$ at periapsis, which corresponds to $7.1 \mathrm{~V} \mathrm{E} / \mathrm{q}$. Another phenomenon is suggested by the observations toward the right-hand portion of Figure 2, where higher energies are observed, with azimuthal components toward antisolar directions. Spacecraft charge, as determined by L. H. Brace (personal communication, 1990), generally does not reach levels that would significantly alter these patterns in Figure 2. For these altitudes, the energy that corresponds to escape from the planet is $8 \mathrm{eV}$ for $\mathrm{O}^{+}$and $1 / 2 \mathrm{eV}$ for $\mathrm{H}^{+}$.

For season 11, although responses below $50 \mathrm{~V} \mathrm{E} / q$ are not available, the fluxes principally seem to be toward the antisolar direction. This season's data are at a substantially higher altitude (peripasis, 2146 to $2205 \mathrm{~km}$ ) than for season 5 and also are from a period nearly 4 years later, when solar activity was lower (see Table 1).

Diagrams analogous to those of Figure 2 have been prepared for each of the other 10 nightside seasons of ion data discussed here. Considering these as representing a sequence of different altitudes (ignoring possible effects due to changing solar activity as the solar cycles progress), the diagrams seem to show a general progression between the two extreme cases of Figure 2, as altitude increases.

Linear correlations of various measures of the escaping, antisunward flowing ions, with various free-stream solar wind parameters, (such as speed, proton number density, dynamic pressure, electric field, measured at other times on the same orbit) have been calculated. A correlation coefficient of +0.43 is obtained for solar wind dynamic pressure, versus the fraction of observations with "low-energy" ion flows in the hemisphere centered on the antisolar direction, for 1355 to $1925 \mathrm{~km}$ periapsis altitudes (seasons 7, 8, 9, and 19). However, since there is no reason known to exclude the observations down to $1050 \mathrm{~km}$ periapsis altitude (seasons 6 and 20), the calculations were extended. We found that inclusion of the additional data results in correlation coefficients of $+0.20,+0.16$, and +0.03 for solar wind proton density, dynamic pressure, and speed, respectively. Some correlation calculations of ion flux against interplanetary electric field and against ion $\mathbf{E} \times \mathbf{B}$ drift speed in the tail region were also done; no significant correlations were found.

Brace et al. [1990] obtained a negative correlation of solar wind dynamic pressure with nightside ionosphere electron density, when periods of low solar activity only were used. The nature of this result is somewhat different from those reported above, in that it refers to somewhat higher altitudes (up to $3000 \mathrm{~km}$ ), and bulk ionosphere densities, rather than escaping fluxes, as well as to lower solar activity.

Correlations between the nightside ion flow directions and the corresponding magnetic field orientations, provided by the spacecraft's magnetometer [Russell et al., 1980], have been studied. One of the results is presented on Figure 3, namely, histograms of the angle between the magnetic field, and the antisolar direction, at times when the low-energy ion fluxes are observed (above a noise threshold), for four nightside periapsis seasons. Here the periapsis altitudes increase, in general, for later seasons. For two of these seasons (6 and 8) the histograms are not consistent with a random distribution of field orientations but rather suggest a tendency for solarantisolar (tailward) alignment of the magnetic fields at times the ion flows are observed. Furthermore, if just a higher energy portion of the observations $(E / q \geq 20 \mathrm{~V})$ for season 6 is selected, the tendency for tailward alignment seems to be increased. For the remaining two seasons ( 5 and 9) the histograms seem more consistent with a random distribution of field orientations.

The aforementioned conclusions can be drawn from values of $\chi^{2} /$ d.f. obtained from the deviations from sine curves whose amplitudes are determined by weighted least squares fits to the histograms of angles. These values are 1.5 and 2.1 for seasons 5 and 9 , respectively, but are 6.9 and 41 for seasons 6 and 8 , respectively. Consequently, data from the latter two seasons are significantly more poorly approximated by a random distribution of angles between the flow and $B$, than are seasons 5 and 9. For the higher-energy data from season 6 , $\chi^{2} /$ d.f. $=16$, which indicates significantly greater $(2 \sigma)$ deviation from a random distribution, than the case of all the data for this season.

Figure 4 gives histograms of the angles, projected into Venus' equatorial plane, between the magnetic field, and the

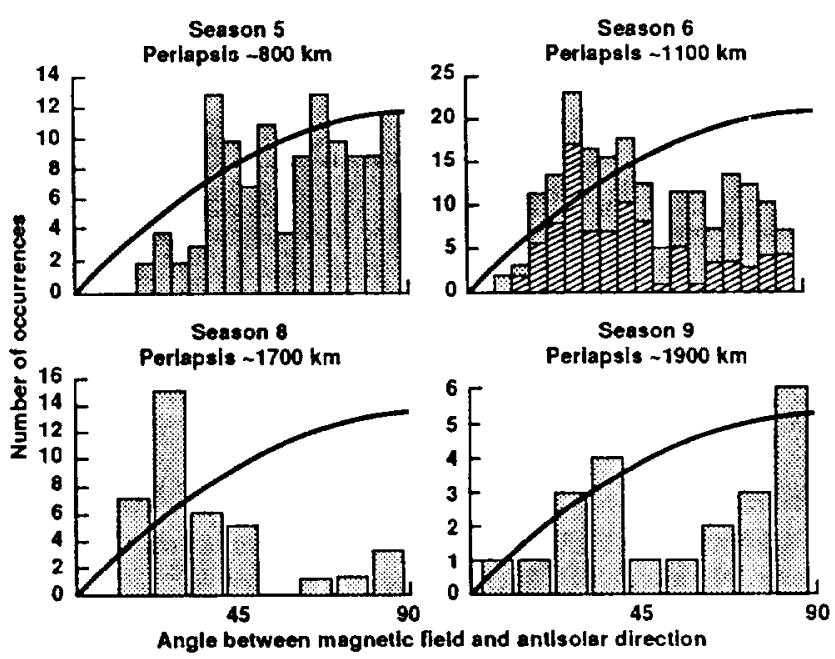

Figure 3. Histograms of the angles between the magnetic field, and the antisolar direction, at times when low-energy ion fluxes were observed, for nightside seasons $5,6,8$, and 9 . The solid (sine) curves indicate the distribution expected for random orientations of the magnetic field. In the case of season 6 , the overlaid histogram represents the results for just the higher energies $(E / q \geq 20 \mathrm{~V})$. 


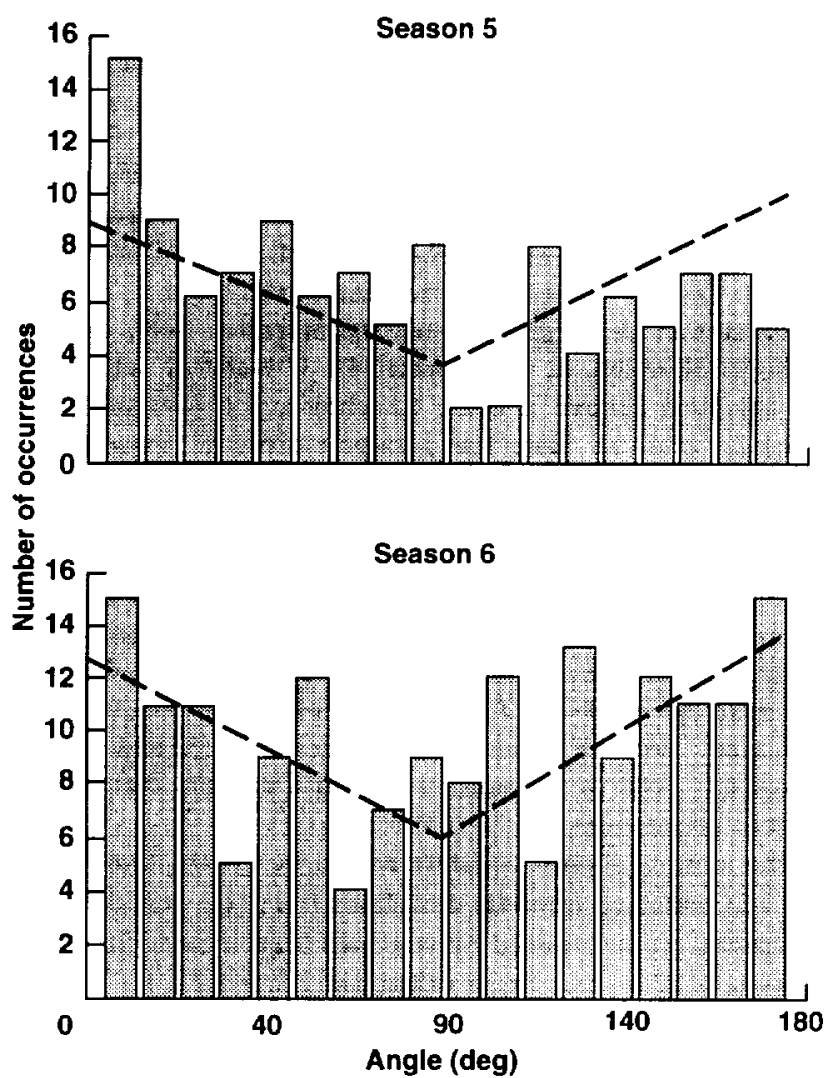

Figure 4. Histograms of the angles between the azimuthal projections onto Venus' equatorial plane, of the magnetic field and the ion flows, for nightside seasons 5 and 6 . Linear least squares fits to the data are shown, also.

ion flows, for two seasons. The correlation between the number of cases, and the angle, was calculated, without distinguishing between coincident and opposing alignments. The correlation was fairly high (correlation coefficient 0.8 ) for season 5, which suggests a tendency for alignment of the field and the flows. The analogous correlation coefficient was only 0.6 for season 6 . The absolute values of the slopes of these fits are $0.11 \pm 0.05$ and $0.14 \pm 0.06$ events per degree of angle, for seasons 5 and 6 , respectively.

In Figure 5, histograms of the "inclination" of the field (cosine of the angle by which the magnetic field is inclined out of Venus' orbital plane), at times when ion fluxes were observed, are given for three seasons, 5, 6, and 11. The season 11 ion data are for a somewhat higher-energy range than for the other two, namely, 50 to $-5000 \mathrm{~V} \mathrm{E} / q$. These three seasons, which correspond to successively higher peripasis altitudes, show increasingly pronounced tendencies for the magnetic fields to lie close to Venus' orbital plane when the flows are observed. This tendency is even more pronounced if, for season 6, attention is restricted to only a higher-energy portion of the data $(E / q \geq 20 \mathrm{~V})$.

For one of the nightside periapsis seasons (season 6), the data, where available, from the Orbiter retarding potential analyzer (ORPA) [Knudsen et al., 1980] and neutral mass spectrometer (ONMS) [Niemann et al., 1980], which has an operating mode that can provide mass-analyzed neasurements of superthermal ions, have been compared with the plasma analyzer (OPA) data, in order to gain clues about the ion species producing the plasma analyzer responses. For season 6 , the largest responses in the plasma analyzer low-energy ion mode occurred on seven different orbits. Corresponding ion densities were available from the retarding potential analyzer (ORPA) for five of the orbits and superthermal ion fluxes [Kasprzak et al., 1982, 1987] from the neutral mass spectrometer (ONMS) for three of the orbits. The spacecraft altitudes during this period are near the upper limit for reliable ion composition measurements by the ORPA [Knudsen et al., 1986]. In Figure 6 are given the ion fluxes from the OPA, at the energies of the peak fluxes, plotted against the corresponding ORPA $\mathrm{H}^{+}$and $\mathrm{O}^{+}$number densities, for the five
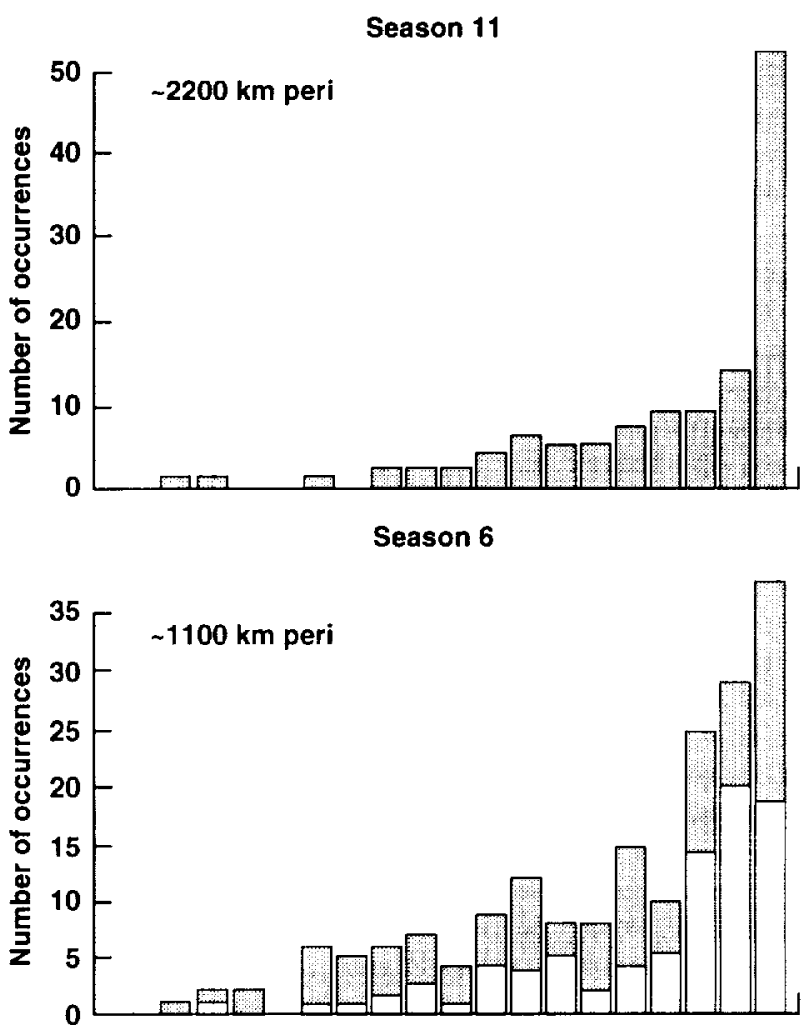

Season 5

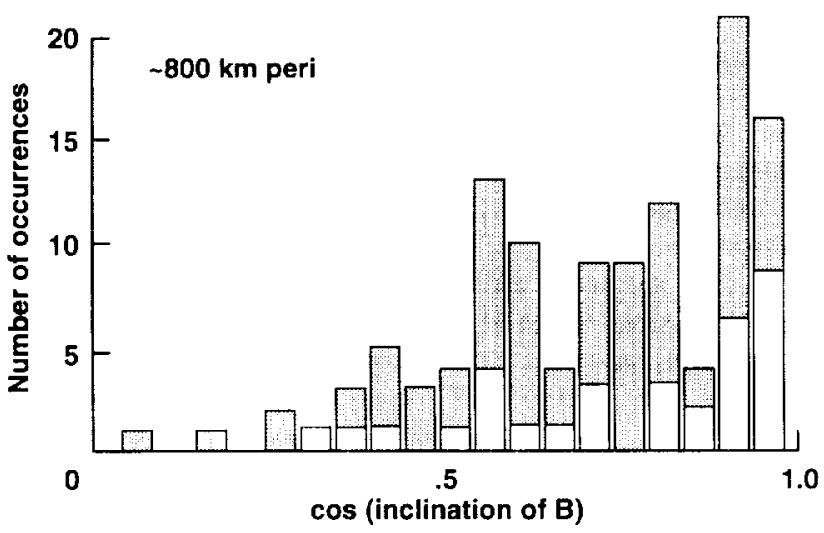

Figure 5. Histograms of the magnetic field "inclination," at times of observation of ion fluxes, for nightside seasons 5, 6, and 11. The overlaid histograms for seasons 5 and 6 give results when lower energies $(E / q<20 \mathrm{~V})$ are excluded. For season 11, the results are only for energies of $50 \mathrm{~V}(E / q)$ and above. 

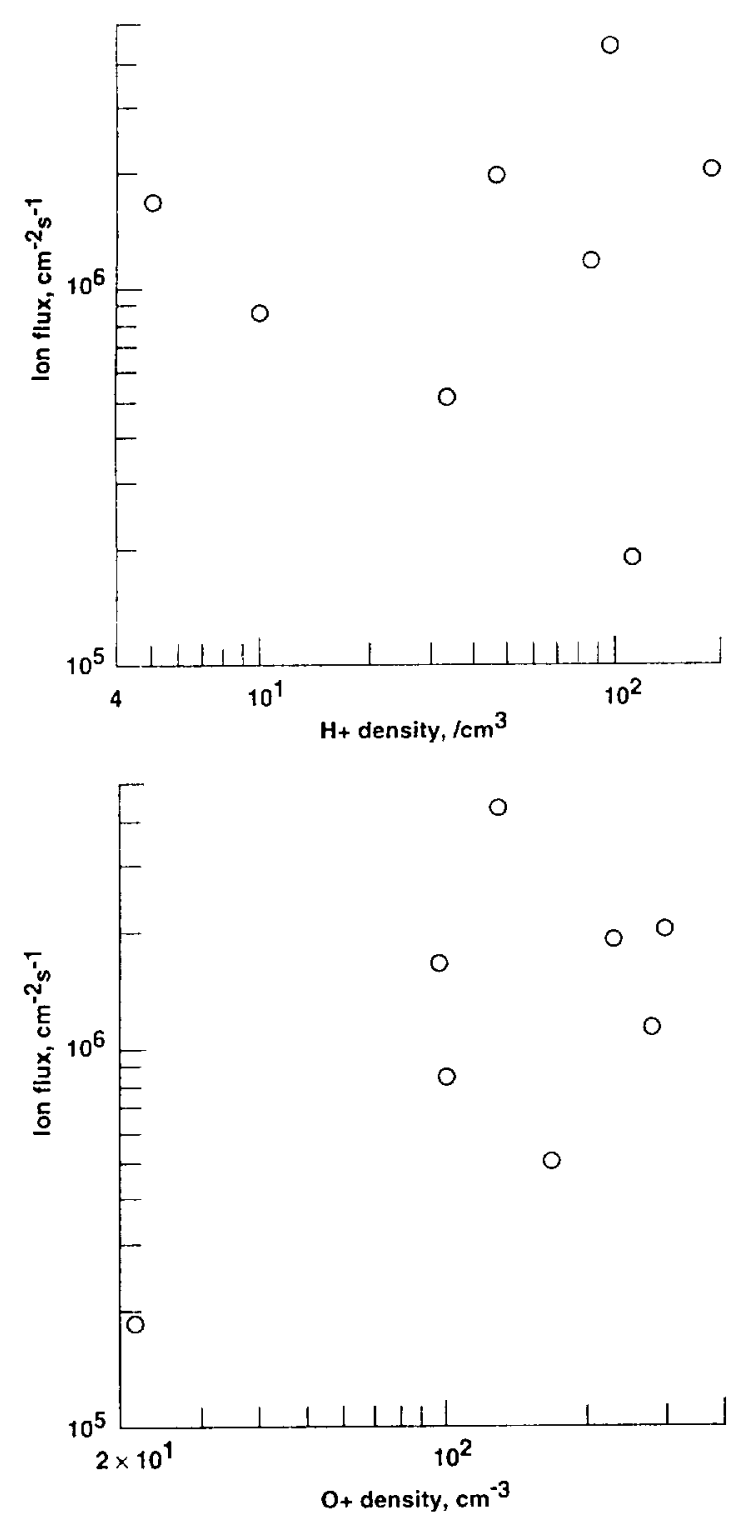

Figure 6. OPA ion fluxes plotted against bulk ionosphere densities of $\mathrm{H}^{+}$and $\mathrm{O}^{+}$, for five orbits during season 6 . Each of these orbits is among the seven of that season with the largest OPA responses, and also with the ORPA data for ionosphere number density. orbits with ORPA ion density data. There is no correlation between the densities of the bulk ionospheric components, given by the ORPA data, and the ion fluxes detected by the OPA.

For one of the three orbits with ONMS superthermal ion data, and a large OPA flux in the low-energy ion mode the peak ONMS responses are near periapsis but are relatively weak. Also, the energy of the largest OPA fluxes was $\sim 15 \mathrm{~V} \mathrm{E} / q$ for that orbit, below an ONMS energy threshold of $-36 \mathrm{eV}$. For another of these three orbits, the largest OPA flux (at $\sim 38 \mathrm{~V}$ $E / q$ ) occurs just $\sim 1 / 2 \mathrm{~min}$ following a peak superthermal $\mathrm{O}^{+}$ flux of $8 \times 10^{6} \mathrm{~cm}^{-2} \mathrm{~s}^{-1}$, consistent with the flux observed by the OPA, $1.2 \times 10^{6} \mathrm{~cm}^{-2} \mathrm{~s}^{-1}$, over a narrower energy range. For the last of these three orbits (orbit 1171), the most intense OPA flux $(-38 \mathrm{~V} \mathrm{E} / q)$ follows a period that began $\sim 4 \mathrm{~min}$ previously, during which superthermal $\mathrm{O}^{+}$fluxes were present, while the OPA was sampling energies below the ONMS threshold. Also, the $\mathrm{H}^{+}$and $\mathrm{O}^{+}$densities are about equal at this time. Later, quite close to the terminator, $\sim 70 \mathrm{~V} \mathrm{E} / q$ fluxes were observed by the OPA; these must be hydrogen.

This comparison of ONMS and OPA data shows that some of the low-energy OPA responses are consistent with superthermal $\mathrm{O}^{+}$fluxes, as measured by the ONMS. The comparison with ORPA data indicates that only a fraction of the bulk ionospheric $\mathrm{O}^{+}$is required to be energized occasionally, if this is the source mechanism for the fluxes detected by the OPA plasma analyzer.

For many of the nightside seasons, estimates of the mean escaping ion flux have been made from the OPA low-energy nightside ion flux measurements, as well as deductions concerning the escaping energy spectrum. Summing all these flux measurements (above a threshold) for a season, and assuming that all the ions are singly charged, yields a mean escaping flux of $<2 \times 10^{6} \mathrm{~cm}^{-2} \mathrm{~s}^{-1}(0-250 \mathrm{~V}$ E/q range). The upper limit is required because all the measurements at threshold flux levels (up to $95 \%$ of the samples at the higher altitudes) were taken to be at the threshold value, whereas it is reasonable that some of those fluxes must have been lower than that.

Some idea of the energy distribution of the escaping ion fluxes can be obtained by accumulating the fluxes in each energy window, for entire nightside seasons, and correcting for the electric potential of the spacecraft when it is significant. The result for season 10 is given in Figure 7,

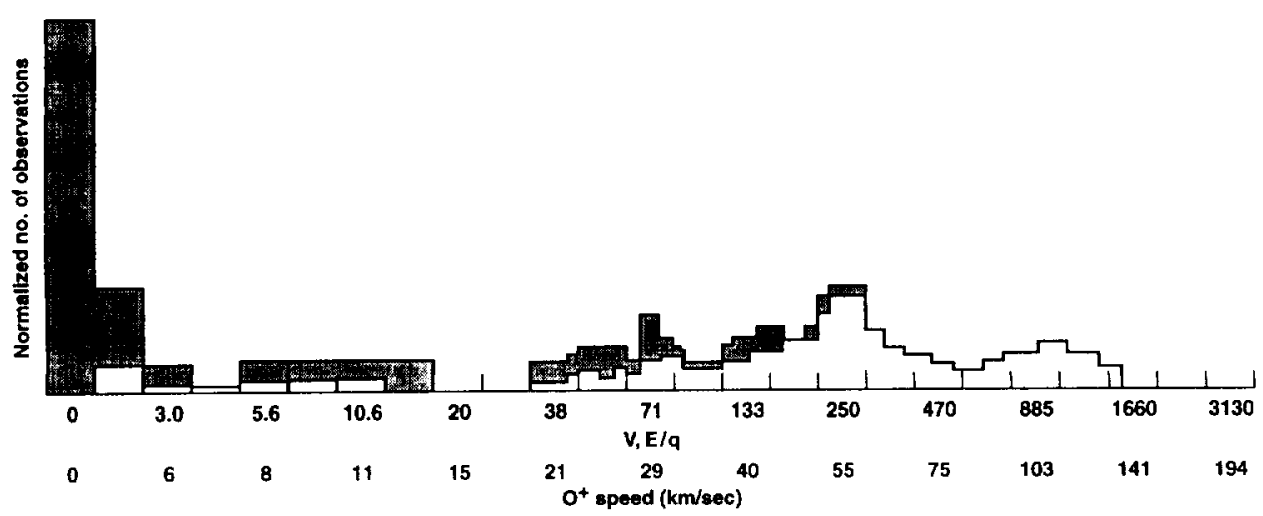

Figure 7. Energy distribution of occurrences, corrected for spacecraft electrostatic potential, of ion fluxes for nightside season 10; the shaded portion is for ion flow toward the planet. The values for 0.25 and $27 \mathrm{~V}$ are low because of an effect of instrumental noise. 
which shows a most probable ion energy of $250 \mathrm{~V} \mathrm{E} / q(55 \mathrm{~km}$ $\mathrm{s}^{-1}$ for $\mathrm{O}^{+}$). The relative amounts of observation at 0 to $250 \mathrm{~V}$ $E / q$ (low-energy mode), and above $50 \mathrm{VE} / q$ only (the other of (wo relevant operating modes of the experiment), had to be accounted for when the Figure was prepared. For periapsis altitudes near $\sim 1100 \mathrm{~km}$, lower than the $\sim 2100 \mathrm{~km}$ level characteristic of season 10 , the most probable escaping ion energies obtained this same way are lower, in the vicinity of $50 \mathrm{VE} / q$. The solar activity was also higher at the times of lower periapses (see Table 1).

\section{Summary}

Episodes of tailward directed, low-energy $(0-250 \mathrm{~V} \mathrm{E} / \mathrm{q})$ ion flows were observed on Venus' nightside during the 14-year lifetime of the Pioneer Venus Orbiter spacecraft. Analysis of the data indicates that at higher altitudes (near $2000 \mathrm{~km}$ ) these episodes make up a greater proportion of the ions observed, compared with near $1000 \mathrm{~km}$ altitude, where the nearly stationary nightside ionosphere can also be detected, as the spacecraft passes through it. The ion energies at higher altitude seem to be higher as well, $250 \mathrm{~V} \mathrm{E} / q$ at $-2000 \mathrm{~km}$. However, the raising and lowering of the spacecraft periapsis, in response to orbital perturbations due to solar gravity put the spacecraft at the highest altitudes when solar activity was low. Kasprzak et al. [1991] have reported that night- side, orbital averaged, superthermal $\mathrm{O}^{+}$fluxes increased with increasing solar activity.

The tailward ion flows did not appear to be correlated with solar wind parameters but tendencies for alignment of the flows with the local magnetic field orientation in the tail and for occurrence of the flows at times of tailward oriented magnetic fields were found. These tendencies for alignment with the magnetic field or with tailward magnetic fields seemed to be greater for higher ion energies. The ion flows were not found to be correlated with bulk densities of constituents of the nightside ionosphere, but a correspondence with superthermal $\mathrm{O}^{+}$was found in several instances. The mean escaping ion flux was $<2 \times 10^{6} \mathrm{~cm}^{-2} \mathrm{~s}^{-1}$.

Acknowledgements. One of us (WCK) wishes to acknowledge the support of NASA grant NAGW-3704.

The Editor thanks P. A. Cloutier and another referee for their assistance in evaluating this paper.

\section{References}

Brace, L. H., and A. J. Kliore, The structure of the Venus ionosphere, Space Sci. Rev., 55, 81, 1991.

Brace, L. H., H. A. Taylor Jr., T. I. Gombosi, A. J. Kliore, W. C. Knudsen, and A. F. Nagy, The ionosphere of Venus: observations and their interpretation, in Venus, edited by D. M. Hunten, L. Colin, T. M. Donahue, and V. I. Moroz, 779, University of Arizona Press, Tucson, 1983.
Brace, L. H., W. T. Kasprzak, H. A. Taylor, R. F. Theis, C. T. Russell, A. Barnes, J. D. Mihalov, and D. M. Hunten, The ionotail of Venus: Its configuration and evidence for ion escape, J. Geophys. Res., 92, 15, 1987.

Brace, L. H., R. F. Theis, and J. D. Mihalov, Response of nightside ionosphere and jonotail of Venus to variations in solar EUV and solar dynamic pressure, J. Geophys. Res., 95, 4075, 1990.

Colin, L., The Pioneer Venus program, J. Geophys. Res., 85, 7575, 1980. Hunten, D. M., T. M. Donahue, J. C. G. Walker, and J. F. Kasting. Escape of atmospheres and loss of water, in Origin and Evolution of Planetary and Satellite Atmospheres, edited by S. K. Atreya, J. B. Pollack, and M. S. Matthews, 386, Univsersity of Arizona Press, Tucson, 1989.

Intriligator, D. S., J. H. Wolfe, and J. D. Mihalov, The Pioneer Venus Orbiter plasma analyzer experiment, IEEE Trans. Geosci. Remote Sens., GE-J8, 39, 1980.

Intriligator, D. S., L. H. Brace, P. A. Cloutier, J. M. Grebowsky, R. E. Hartle, W. T. Kasprzak, W. C. Knudsen, and R. J. Strangeway, Evidence for ion transport and molecular ion dominance in the Venus ionotail, J. Geophys. Res., 99, 17,413, 1994.

Kasprzak, W. T., H. A. Taylor, L. H. Brace, H. B. Niemann, and F. L. Scarf, Observations of energetic ions near the Venus ionopause, Planet. Space Sci., 30, 1107, 1982.

Kasprzak. W. T., H. B. Niemann, and P. Mahaffy, Observations of energetic ions on the nightside of Venus, J. Geophys. Res., 92, 291, 1987.

Kasprzak, W. T., J. M. Grebowsky, H. B. Niemann, and L. H. Brace, Superhermal $>36-\mathrm{eV}$ ions observed in the near-tail region of Venus by the Pioneer Venus Orbiter neutral mass spectrometer, J. Geophys. Res. 96. 11.175, 1991.

Knudsen, W. C.. K. Spenner, J. Bakke, and V. Novak, Pioneer Venus Orbiter planar retarding potential analyzer plasma experiment, $I E E E$ Trans. Geosci. Remote Sens., GE.18, 54, 1980.

Knudsen, W. C., K. L. Miller, and K. Spenner, Median density altitude profiles of the major ions in the central nightside Venus ionosphere, J. Geophys. Res., 91, 11,936, 1986.

Knudsen, W. C., A. J. Kliore, and R. C. Whitten, Solar cycle changes in the ionization sources of the nightside Venus ionosphere, J. Geophys. Res., 92, 13,391, 1987.

Niemann, H. B., J. R. Booth, J. E. Cooley, R. E. Hartle, W. T. Kasprzak, N. W. Spencer, S. H. Way, D. M. Hunten, and G. R. Carignan, Pioneer Venus Orbiter neutral gas mass spectrometer experiment, IEEE Trans. Geosci. Remote Sens., GE-18, 60, 1980.

Phillips. J. L.. and D. J. McComas, The magnetosheath and magnetotail of Venus, Space Sci. Rev., 55, 1, 1991.

Russell, C. T., R. C. Snare, J. D. Means, and R. C. Elphic, Pioneer Venus Orbiter fluxgate magnetometer, IEEE Trans. Geosci. Remote Sens., $G E-18,32,1980$.

Strangeway, R. J., The Pioneer Venus Orbiter entry phase, Geophys. Res. Letr., 20, 2715, 1993.

W. T. Kasprzak, NASA-Goddard Space Flight Center, Greenbelt, MD 20771. (e-mail: kasprzak@gsfc.nasa.gov)

W. C. Knudsen, Knudsen Geophysical Research, Monte Sereno, CA 95030.

J. D. Mihalov. Planetary Systems Branch. Mail Stop 245-3, NASAAmes Research Center, Moffell Field, CA 94035-1000. (e-mail: mihalov@windee.arc.nasa.gov)

C. T. Russell, Institute for Geophysics and Planetary Physics, University of California, Los Angeles, CA 90024. (e-mail: ctrussel]@igpp.ucla.edu)

(Received February 21, 1995; revised April 24, 1995 ; accepted April 25, 1995.) 